\title{
Childhood intussusception: the safety case
}

\author{
William E. Shiels II
}

Received: 6 December 2012 / Accepted: 11 January 2013 / Published online: 3 February 2013

(C) Springer-Verlag Berlin Heidelberg 2013

"In any situation, the best thing you can do is the right thing; the next best thing you can do is the wrong thing; the worst thing you can do is nothing."-Theodore Roosevelt

In this issue of Pediatric Radiology, Drs. Fallon et al. [1], as authors of the clinical study titled "Needle decompression to avoid tension pneumoperitoneum and hemodynamic compromise after pneumatic reduction of pediatric intussusception" are to be congratulated for providing a simple, well-articulated and focused report that describes the successful outcomes (with no fatalities) of needle decompression of tension pneumoperitoneum occurring during childhood intussusception reduction. More important than a sterile scientific report, this report provides a critical summary of excellent outcomes that can be predicted by implementing a prospective protocol, with a plan of action that anticipates risk and complications, and responsible acts that result in life-saving management, averting catastrophe and death.

In paraphrased outline form, the authors recommend the following:

1. Prospective definition and understanding of the risk and "worst-case scenario"

2. Prospective protocol for management of risk

3. Outline of a detailed plan of action when risk is realized

4. Anticipatory will to act

5. Evaluation and accountability after the plan is implemented

W. E. Shiels II $(\square)$

Nationwide Children's Hospital, 700 Children's Drive,

Room K140,

Columbus, OH 43205, USA

e-mail: william.shiels@nationwidechildrens.org
In this very practical and technically elegant report, the authors outline the concepts inherent in what is referred to in the United Kingdom (UK) offshore energy industry as a "safety case" [2]. A safety case is a document required by UK legislation that is produced by an operator of a facility that identifies the hazards and risks, describes how the risks are controlled, and defines the safety management system in place to ensure the controls are effectively and consistently applied.

The author's radiology department has incorporated a detailed safety case for childhood intussusception training and clinical care since 2010. In this commentary, I will describe the components and underlying principles of a safety case and detail our department's implementation of the intussusception safety case. A critical principle of a safety case is that the operator takes ownership and responsibility for its successful implementation. The principles of ownership, responsibility and accountability readily apply to the radiologist (the operator) during an air intussusception reduction procedure. My discussion is within the context of my role as a department chairman, fellowship director, resident trainer, clinical radiologist and expert on intussusception who has served as a legal expert witness and consultant in cases of death during air intussusception reduction when a radiologist failed to provide needle decompression of pneumoperitoneum.

The childhood intussusception safety case elements are as follows:

1. The intussusception safety case is defined by the department chairman and GI procedures section chief. When a radiologist engages in the care of a child with intussusception he or she accepts ownership, responsibility and accountability for the intussusception safety case (management of the worst- 
case scenario). The principle here is that the radiologists who create the risk must manage it. It is the radiologist's responsibility to assess care processes, procedures and systems to identify and evaluate the risks during air intussusception reduction and implement the appropriate safety measures, because the radiologist has the greatest indepth knowledge of their patient, procedural capabilities, operating team (especially when fellows and residents are involved) and safety controls.

2. The intussusception safety case must identify the safety-critical aspects of the procedure, both technical and managerial. Analyses of disasters and deaths during air enema reduction almost always show a combination of technical and managerial flaws, which have led to death during air intussusception reduction procedures.

3. Appropriate performance standards must be defined for the operation of the safety-critical aspects of air intussusception reduction, as a matter of protocol, to prevent avoidable morbidity and death. A performance standard encompasses radiologist (and procedure team), performance, items of equipment and procedures (including training) that are used for managing procedural risk. These include detailed performance expectations during air intussusception reduction including surgical consultation, written informed consent, venous access, parental presence, anal seal, patient monitoring (including heart rate and oxygen saturation), colonic insufflation technique, colonic pressure monitoring, detection of perforation and pneumoperitoneum, 18-G needle for decompression, needle decompression technique, and patient management (with handoff) following needle decompression.

4. The radiology care team must be involved. The involvement of all physicians and staff is necessary so they know what happens in practice and why during an air intussusception reduction. This makes it more likely that they will do the right thing because they understand why perforation with intussusception reduction occurs, why death occurs with tension pneumoperitoneum, and their responsibility for active risk management during air intussusception reduction.

5. The safety case is produced in the knowledge that it will be scrutinized by a competent and independent regulator. The department chairman, GI procedures section chief and hospital safety team (also potentially regulatory agencies) assess the intussusception safety case and "accept" the safety case if they are satisfied that the processes demonstrate that the risks of air intussusception reduction will be reduced to as low as reasonably practicable. Once accepted, oversight is provided by the chairman (or appropriate designee) who visits the fluoroscopy suite, interviews radiologists and reviews the procedure logs to monitor the application of the intussusception safety case in practice.

At Nationwide Children's Hospital Radiology Department the implementation of the intussusception safety case starts with children suspected of having intussusception who undergo clinical evaluation by the Emergency Department physicians, including appropriate plain radiographic and sonographic examinations. Once intussusception is diagnosed, surgical consultation is obtained. Surgical and Emergency Medicine physicians are responsible for fluid resuscitation if needed prior to radiology consultation for air intussusception reduction. The radiologist obtains written informed parental consent, including discussion of the potential for intestinal perforation and subsequent needle decompression management (and subsequent operative management). Children are to arrive in Radiology with venous access, pulse oximetry monitor and a nurse for patient monitoring. Children are not sedated for reduction procedures. Prior to beginning the procedure, an $18-\mathrm{G}$ needle (with or without an angiocatheter sheath) is placed in a conspicuous location for ready access and use. Effective anal seal is obtained (external anal gasket seal or rectal balloon seal). Colonic air insufflation commences in a stepwise fashion, progressing from 60 to 80,100 , and finally $120 \mathrm{mmHg}$, with regular colonic pressure monitoring, especially between Valsalva maneuvers. Accurate pressure monitoring (with documentation of maximum insufflation pressure) is performed in between Valsalva maneuvers. If the intussusception is not successfully reduced after 3-5 attempts, 3-5 min in duration each, reduction attempts are discontinued, followed by surgical consultation. Surgical consultation includes discussion of further management including 2- to 4-h delay with further reduction attempts, increasing pressure threshold (with clear understanding of increasing likelihood of intestinal perforation), and transfer of the patient to the operating suite for open intussusception reduction or bowel resection.

Manometric signs of intestinal perforation include acute loss of insufflation pressure with an effective anal seal. Manometric concern for intestinal perforation is followed by fluoroscopic evaluation for pneumoperitoneum. Supine crosstable lateral abdominal radiograph might be obtained if fluoroscopic evaluation is inconclusive. Management of intestinal perforation also includes movement of the fluoroscopic tower and rapid clinical evaluation of the patient for signs of respiratory distress, mental status changes, decreasing oxygen saturation, tachycardia and hypotension. If intestinal perforation is confirmed, and abdominal wall tension is identified, betadine or chloraseptic solution is applied to the anterior abdominal wall and an 18-G needle is inserted into the linea alba (midline) in a supraumbilical location for immediate peritoneal decompression. The needle or sheath is left in place until stable vital signs 
are confirmed and there is less abdominal wall tension. If surgical team members are not present in the fluoroscopic suite, the Emergency Department physician is notified of the intestinal perforation and resuscitation details, with clear clinical handoff information relayed to Emergency Department physician and nursing personnel. Following this, the attending surgeon is called with relay of details regarding the reduction procedure, intestinal perforation and resuscitation.

\section{Actual case: Fall 2012}

A 5-month-old boy presented with 4 days of intermittent abdominal pain, bloody stools, abdominal distention and bilious vomiting. Plain radiographs demonstrated evidence of small-bowel obstruction. Abdominal US examination confirmed the diagnosis of ileocolic intussusception. Surgical consultation was obtained and the radiologist was consulted for air intussusception reduction, following the Department of Radiology air intussusception reduction procedure protocol. Prior to reduction, the boy's heart rate was $140 \mathrm{bpm}$, oxygen saturation $100 \%$ on room air and blood pressure 101/73 mmHg. During the procedure, the intussusception was not reducible beyond the ileocecal valve and there was concern for intestinal perforation. The boy was clinically stable. Review of fluoroscopic images on the inroom monitors was inconclusive for pneumoperitoneum, so images were sent to PACS for review, which confirmed pneumoperitoneum. Following PACS image review, the radiologist evaluated the child and found him to be listless, semi-conscious, with a heart rate of $184 \mathrm{bpm}$, blood pressure of $87 / 46 \mathrm{mmHg}$, and a tense abdomen. The radiologist immediately prepped the abdominal wall and inserted the 18-G needle into the linea alba, supraumbilical location, with an immediate hissing sound with decompression of the pneumoperitoneum. Within $30 \mathrm{~s}$ of needle decompression the boy was alert, was visually tracking his parents with appropriate responses, and had a blood pressure of 117/86 $\mathrm{mmHg}$ and heart rate of $135 \mathrm{bpm}$. The radiologist's attention to procedure protocol, recognition of intestinal perforation, accurate and rapid assessment of the boy's deterioration, and prompt and appropriate intervention saved this child's life.

The study by Fallon et al. [1] is a critical foundational report that illustrates the importance of clear protocols, prospective procedural risk identification (worst-case scenario), evidence basis for well-rehearsed action plans, the need for willful action (needle decompression of pneumoperitoneum), and clear radiologist responsibility for procedural risk management. The intussusception safety case is simple and clear for articulation, easily taught and readily reviewed prior to beginning each procedure. In a onesentence summary, a radiologist following the intussusception safety case approach anticipates the worst-case scenario, readily identifies the risk of tension pneumoperitoneum, has rehearsed a management plan for the worst-case scenario and calmly acts to decompress the tension pneumoperitoneum, preventing the worst-case scenario of death as an avoidable outcome.

\section{References}

1. Fallon SC, Kim ES, Naik-Mathuria BJ et al (2013) Needle decompression to avoid tension pneumoperitoneum and hemodynamic compromise after pneumatic reduction of pediatric intussusception. Pediatr Radiol. doi:10.1007/s00247-012-2604-y

2. United Kingdom Statutory Instrument 2005 No. 3117, The Offshore Installations (Safety Case) Regulations 2005. Available via http:// www.legislation.gov.uk/uksi/2005/3117/contents/made Accessed 11 January 2013 